\title{
Bandwidth Allocation Scheduling Algorithms for IEEE 802.16 WiMax Protocol to Improve QoS: A Survey
}

\author{
Avinash Kaur \\ Student \\ Department of Computer \\ Science \& Engineering \\ S.K.I.E.T Engineering College, \\ Kurukshetra, Haryana, India
}

\author{
Harvinder Singh \\ Assistant Professor \\ Department of Computer \\ Science \& Engineering \\ H.C.T.M Technical Campus, \\ Kaithal, Haryana, India
}

\author{
Parveen Sharma \\ Associate Professor \& HOD \\ Department of Computer \\ Science \& Engineering \\ S.K.I.E.T Engineering College, \\ Kurukshetra, Haryana, India
}

\begin{abstract}
In recent times, wireless network is extensively accessed technology to connect remote user terminal with its primary network. QoS is the vital parameter that can be treated wisely when data is transferred between terminal users and network administrators. In WiMax, QoS is determined accurately at MAC layer but bandwidth allocation scheduling algorithm which defines QoS is not clearly defined in IEEE 802.16 network architecture. This paper evaluates and compare various existing algorithms and enlighten different issues in designing of these algorithms, furthermore a new bandwidth allocation scheduling algorithm is proposed for IEEE 802.16 WiMax protocol in order to improve Quality of Service (QoS).
\end{abstract}

\section{Keywords}

WiMax, Schedule, QoS, Bandwidth Allocation Scheduling, IEEE 802.16

\section{INTRODUCTION}

WiMax (World Wide Interoperability for Microwave Access) is a telecommunication technology designed to provide effective transmission of data using different modes of transmission like mesh and PMP (Point to multipoint). WiMax is a high performance end to end network protocol. Its features are increased data rate, high performance, fair QoS, highly secured communication of data with less packet delay. There are two main types of WiMax services: mobile and fixed. Mobile WiMax enables users to access internet while travelling whereas fixed WiMax stations provide wireless internet access to clients within a fixed radius. So concept of WiMax is introduced to increase the range of network. The basic WiMax IEEE 802.16 architecture consists of Base Station (BS) and Subscriber Station (SS). BS acts as a central entity to transfer all the data from SS in a PMP mode. Transmissions take place through two independent channels: Downlink channel (from BS to SS) and Uplink channel (from SS to BS). Uplink channel is shared by all SSs while
Downlink channel is used by BS. The standard IEEE 802.16 supports four different flow classes of QoS and the MAC layer supports a request-grant mechanism for data transmission in uplink direction. This standard does not define a slot allocation criterion and scheduling architecture for any type of service. A scheduling module is necessary to provide QoS for each class. IEEE 802.16 defines the following four types of service flow with distinct QoS requirements. These are as follows:

Unsolicited Grant Services (UGS) designed to support Constant Bit Rate (CBR) services such as voice applications.

Real-Time Polling Services (RTPS): designed to support real-time services that generate variable size data packets, such as MPEG video.

Non-Real-Time Polling Services (NRTPS): designed to support non-real-time and delay tolerant services that require variable grant burst type data on a regular basis such as FTP.

Extended Real Time Polling Services (ERTPS): designed to support real time applications with variable data rate which require guaranteed data and packet delay.

Best Effort (BE): designed to support data streams that do not require any guarantee in QoS such as HTTP.

\subsection{WiMax Network Architecture}

The network reference model envisions unified network architecture for supporting fixed, nomadic, and mobile deployments. It is based on an IP service model. Below is simplified illustration of an IP - based WiMax network architecture. The overall network is logically divided into three parts- Mobile Stations (MS), Access Service Network

(ASN) and Connectivity Service Network (CSN). The network reference model defines a number of functional entities and interfaces between those entities like Base Station (BS), Access Service Network Gateway (ASN-GW), Connectivity Service Network (CSN) as shown in Figure 1 :- 


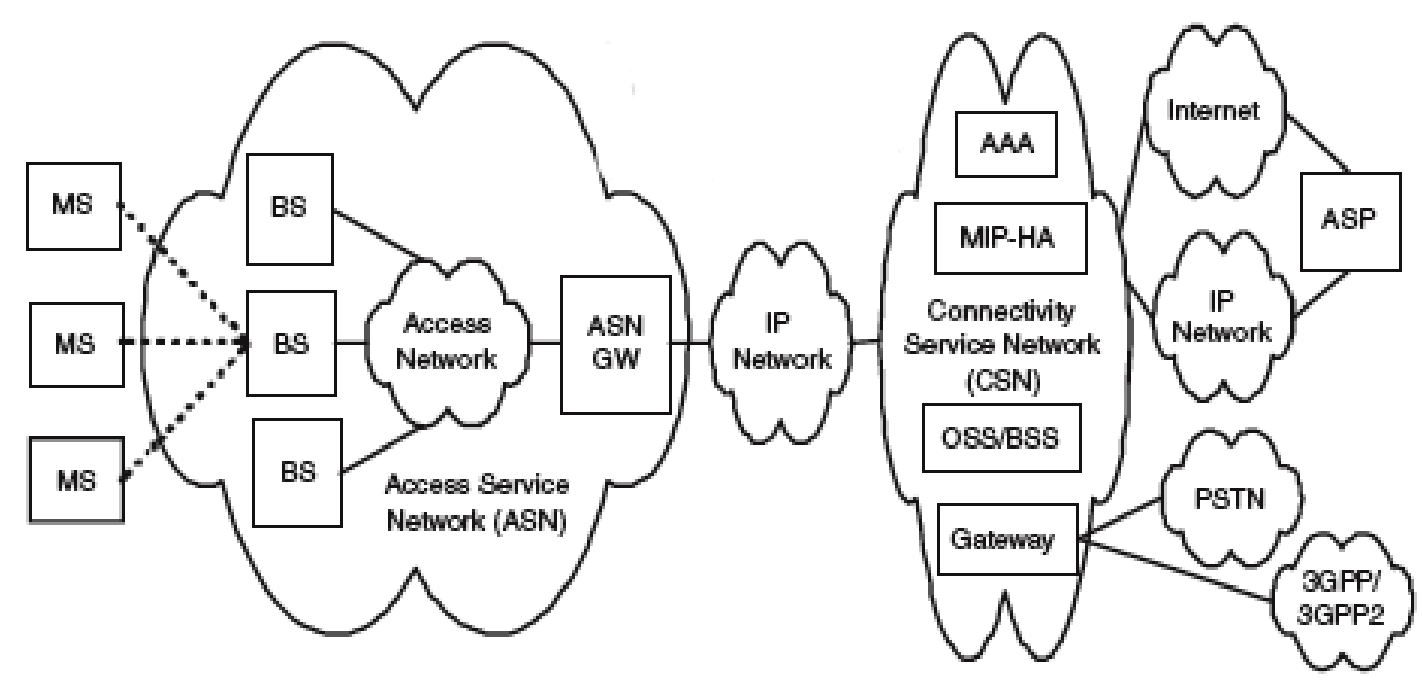

Fig. 1: IP-Based WiMax Network Architecture[16]

\section{RELATED WORK}

Qingwen Liu, Shengli Zhou and Georgious B.Glannakis [3] proposed a cross layer scheduling design for multiuser scheduling at Data Link Layer. Every user is compatible with the AMC (Adaptive Modulation Control) at physical layer which intern is compatible with even worst complexity scheduling algorithms. It has high scalability and gives average performance analysis. Fixed buffer length helps in allocation of bandwidth to resources which improve QoS.

Jamshed Hasan [4] develops a new technology which is based on threats, risk and vulnerabilities in real situations. He represents the security problems of IEEE 802.16standard and his main focus is on security constraints, threats and vulnerabilities and risks associated with this standard.

Jianfeng Song Jiandong Li Changle Li [7] proposed a cross layer scheduling algorithm based on genetic algorithm (CLSAGA) under the Network Utility Maximization (NUM) which allocates resources for each service flow. It improves Signal Noise Ratio (SNR) and increase bandwidth to different users to get higher QoS.

Ronak Farhadi, Vahid Tabataba Vakili, Shahriar Shirvani Moghada [10] reviewed that scheduling is an essential component in WiMax network for efficient use of radio resources. This algorithm considers QoS and channel condition of wireless network. CACBQ algorithm for its scheduling decisions not only considers QoS of each channel but also channel quality parameter. CACBQ algorithm has the best value in delay and packet loss rate for real time services. MAX-SNR algorithm has best throughput.

\section{ANALYSIS AND COMPARISON OF COMMONLY USED SCHEDULING ALGORITHMS}

\subsection{Scheduling Algorithms}

The basic WiMax structure consists of one Base Station (BS) and one or several Subscriber Station (SS). The communication flow from BS to SS is called Down-Link (DL) traffic while from SS to BS is called Up-Link (UL) traffic. Various types of scheduling algorithms and their comparison are discussed below:

\section{2 (WRR) Weighted Round Robin}

It is a work-conserving algorithm in which it will continue allocating bandwidth to the SS as long as they have backlogged packets. The WRR algorithm assigns weights to each SS and the bandwidth is then allocated according to the weights. The algorithm will not provide good performance in the presence of variable size packets because the bandwidth is assigned according to the weights only [5]. Weight to SS can be calculated by the formulae. Weight, $w_{i}=\frac{r_{i}}{\sum r_{i}} \quad\left(r_{i}=\right.$ min reserved traffic rate)

Working of Weighted Round Robin algorithm is shown in Figure 2: 


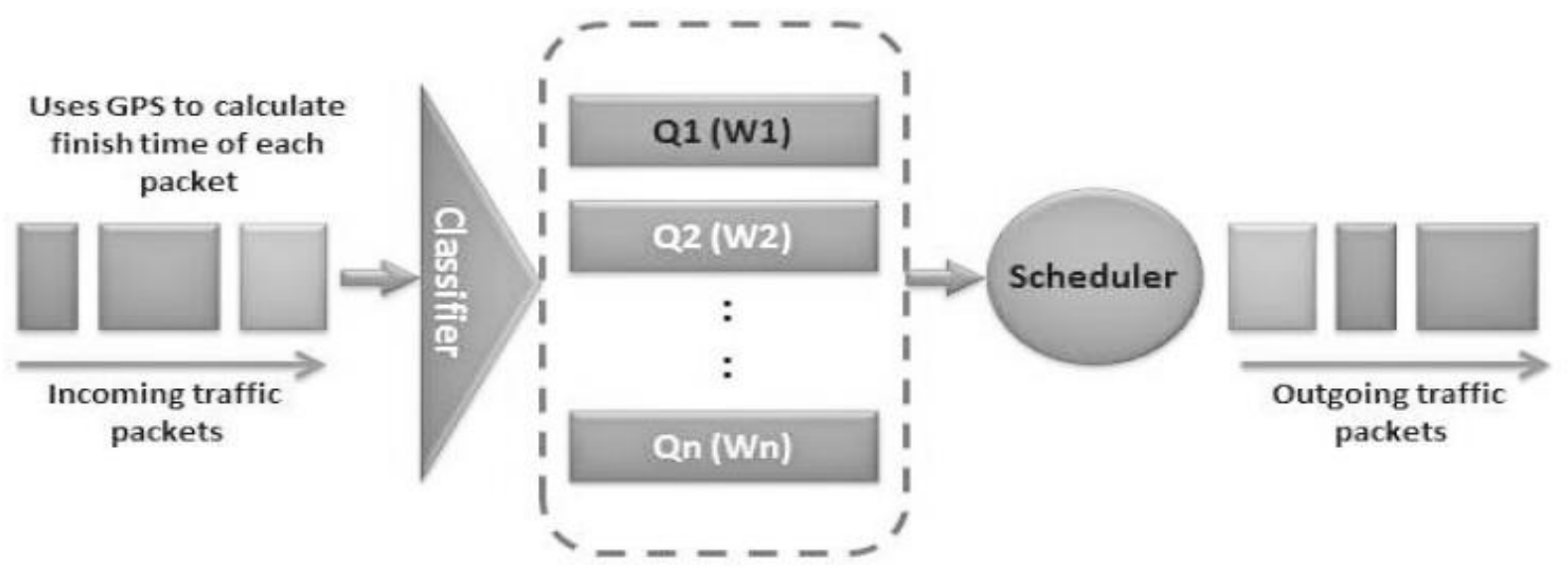

Fig. 2: Weighted Round Robin [5]

\subsection{CIF-Q (Channel Condition Independent Packet Fair Queue)}

This algorithm is complex as compare to other algorithms because in actual system, amount of services which are received and referenced need to be secure and updated. It uses start time fair queuing for error free systems which improve QoS, throughput and packet delay. At uplink stage it calculates the virtual time period of data packets from host to receiver [8]

\subsection{CSDPS (Channel State Dependent Packet Scheduling Algorithm)}

This algorithm ensures higher throughput and fair distribution of data packets for wireless network. It adopts changes of network state which are caused by time and location of the variable host. This algorithm cannot be used for mobile networks because it does not give fair bandwidth and QoS for these networks [8].To solve this problem, a scheduling algorithm [10] that combines CSDPS with CBQ (Class Based Queue) is proposed. The model CSDPS+CBQ gives higher throughput and equal distribution of packets on wireless network. But it does not recommend any mechanism, which calculates the bandwidth compensation, if the network suffers from previous error connection state.

\subsection{I-CSDPS (Improved Channel State Dependent Packet Scheduling)}

This algorithm deals with the length of packets variably. It uses DRR (Deficit Round Robin) algorithm and accurate bandwidth compensation counter which deals with the data packet automatically and increase the flexibility and throughput of the network. There is no effect on the flow of data if network receives some additional services [8].

\subsection{SBFA (Server Based Fair Approach)}

This algorithm has very simple architecture and provides a framework which is integrated with wired network architecture. This algorithm provides bandwidth compensation in data flow which occurs due to network error. At receiver, packets are suffered from loss and cannot be recovered because transmitted channel has errors. Then it creates virtual slots and shares their slots in LIFS. LIFS is limited by service rate and does not receive additional services [15].

\subsection{WFQ (Weighted Fair Queuing)}

It is a packet-based approximation of the Generalized Processor Sharing (GPS) algorithm. GPS is an idealized algorithm that assumes a packet can be divided into bits and each bit can be scheduled separately. The WFQ algorithm results in superior performance compared to the WRR algorithm in the presence of variable size packets. The finish time of a packet is essentially the time of the packet that would have finished service under the GPS algorithm. The disadvantage of the WFQ algorithm is that service packet wouldn't have started service under the GPS algorithm because the WFQ algorithm does not consider the start time of a packet [5]. Working of Weighted Fair Queuing algorithm is shown in Figure 3: 


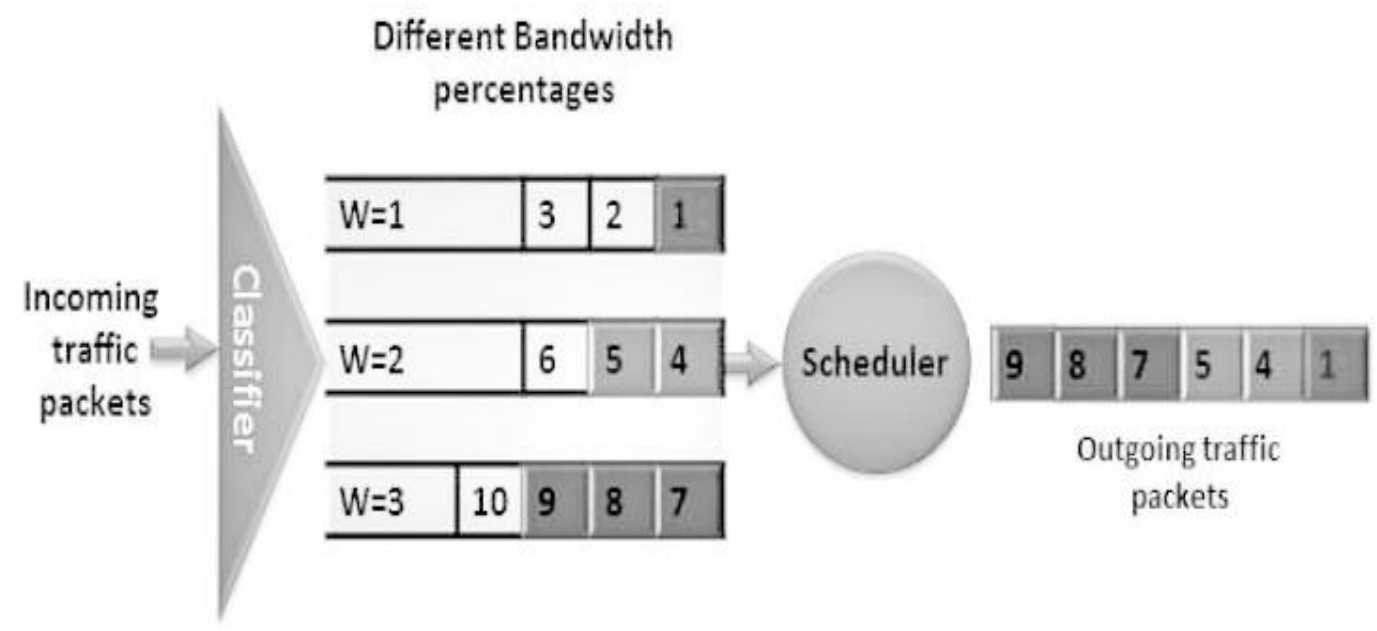

Fig. 3: Weighted Fair Queuing [5]

\subsection{PFS (Packet Fair Scheduling)}

Essential goals of this packet scheduling scheme are to enhance the system throughput as well as provide fairness among the queues under consideration. Although PFS is simple and efficient, it cannot guarantee any QoS requirement such as delay and delay jitter due to its original design for saturated queues with non real-time data service. A scheduling P is "proportionally fair" if and only if, for any feasible scheduling.

$$
\sum_{u} \frac{R_{i}^{(\mathrm{s})}-R_{i}^{(\mathrm{p})}}{R_{i}^{(\mathrm{p})}} \leq 0
$$

Where $\mathrm{U}$ is the user set, $R_{i}^{(\mathrm{s})}$ and $R_{i}^{(\mathrm{p})}$ are the average rates of users i by scheduler $\mathrm{S}$ and scheduler $\mathrm{P}$, respectively. The PFS scheduler is designed to take advantage of multiuser diversity while maintaining comparable long-term throughput for all users. Let $\mathrm{Rk}(\mathrm{t})$ denote the instantaneous data rate that user $\mathrm{k}$ can achieve at time $\mathrm{t}$, and let $\mathrm{Tk}(\mathrm{t})$ be the average throughput for user $\mathrm{k}$ up to time slot t. The PFS scheduler selects the user, denoted as $\mathrm{k}^{*}$, with the highest $\operatorname{Rk}(\mathrm{t}) / \operatorname{Tk}(\mathrm{t})$ for transmission. In the long term, this is equivalent to selecting the user with the highest instantaneous rate relative to its mean rate [15].

\subsection{Integrated Cross-layer Scheduling}

Both RR (Round Robin) and PF (Proportional Fairness) cannot manage the resource allocation and grants an appropriate QoS per connection. The ICLS scheduler is based on a priority function for each queue, where the priority metric of each queue is updated according to its service status and channel condition in the physical layer [3]. Thus, the scheduler can provide the prescribed diverse QoS guarantees. This scheduler is hard to be practically deployed due to its high implementation complexity [7].

\subsection{CLSAGA (Cross Layer Scheduling Algorithm based on Genetic Algorithm)}

This algorithm is based on Genetic Algorithm (CLSAGA) under the Network Utility Maximization (NUM) proposed to allocate resources for each service flow. The real coded genetic algorithm is employed to solve NUM optimization problem. Adaptive modulation coding scheme (AMC) and QoS category index of each service flow jointly decide the weights of utility functions to calculate the scheduling scheme of medium access control (MAC) layer. Simulation results show that this approach guarantees the QoS requirements and balances priorities of the mobile stations [7].

\subsection{TCP-aware Uplink Scheduling}

This scheduling algorithm manages the BE traffic because it is difficult to estimate the amount of required bandwidth due to dynamic changes in the sending rate of traffic. The sending rate of a TCP flow is changed over time due to the AIMD (Additive Increase Multiplicative Decrease) feature in a shortterm period and available bandwidth in a long-term period. The objective of the proposed scheme is to realize the maxmin fairness in bandwidth allocation among BE flows while maintaining high link utilization. The scheme measures the sending rate of each flow and allocates bandwidth based on the measured sending rate [10].

\subsection{Comparison of the algorithms}

Comparisons among different scheduling algorithms with different parameters are specified in table1: 
Table1. Comparison between different bandwidth allocation scheduling algorithms for WiMax IEEE 802.16 (with notations B - Best, A - Average, W - Worst)

\begin{tabular}{|c|c|c|c|c|c|}
\hline Algo & QoS & $\begin{array}{c}\text { Load } \\
\text { Balancing }\end{array}$ & $\begin{array}{c}\text { Delay for ERTPS } \\
(\mathbf{m s})\end{array}$ & $\begin{array}{c}\text { Packet Loss Rate for } \\
\text { RTPS (Mbps) }\end{array}$ & $\begin{array}{c}\text { Through-put for } \\
\text { RTPS (Mbps) }\end{array}$ \\
\hline WRR & B & B & A & W & A \\
\hline CIF-Q & B & A & A & A & A \\
\hline CSDPS & A & A & W & A & A \\
\hline I-CSDPS & B & A & A & W \\
\hline SBFA & A & W & W & B \\
\hline WFQ & A & B & A & A & B \\
\hline PFS & B & B & A & A & B \\
\hline CLSAGA & B & B & A & A & B \\
\hline ICLS & B & B & A & A & A \\
\hline TCP-US & B & B & & & A \\
\hline
\end{tabular}

\section{BANDWIDTH ALLOCATION SCHEDULING ALGORITHM FOR WIMAX PROTOCOL}

In IEEE 802.16 WiMax architecture, there are four different data service types- UGS, RTPS, NRTPS, BE. Every algorithm has its adequacies and deficiencies when compared on the basis of different parameters. CSDPS+CBQ algorithm fulfill all requirements of IEEE 802.16 protocol in terms of throughput and QoS whereas it does not provide equal bandwidth compensation. I-CSDPS cannot balance load equally. PFS and RR do not provide a guaranteed QoS per connection. So these algorithms are not specially designed for IEEE 802.16 WiMax architecture. As per the need of IEEE 802.16 network architecture, a new algorithm is proposed which removes the numerous drawbacks and enhances the QoS for WiMax protocol.

\subsection{Design Issues}

While reviewing the above algorithms, found some major consequences in design of IEEE 802.16 network, which are as below:

\subsubsection{The fairness of the bandwidth allocation between different nodes}

Bandwidth varies according to the requirement of channel so a data terminal carries different types of data transmission modes. A max-min fairness allocation is achieved when bandwidth is allocated equally at different stations. Bandwidth utilization cannot be appropriate because data terminals change their requirements accordingly.

\subsubsection{Data transmission delay}

It is also known as the packet delay and it is the total time required to push all packets at their destination. We can calculate the data transmission delay by the following formula:

$\mathbf{D}_{\mathrm{T}}=\mathbf{N} / \mathbf{R}$ Seconds

$\mathrm{D}=$ Data Transmission delay in seconds.

$\mathrm{N}=$ Number of bits per second.

$\mathrm{R}=$ Rate of transmission (bits per second).

\subsubsection{Data throughput of the system}

It is the amount of work that a node can complete in given time period. In throughput, it checks overall performance of the system, speed and workload. There are some factors that determine the throughput:

1. Type of data being transferred.

2. Number of users on the network.

3. Network topology.

4. User and server computer.

4. Internetworking devices. 


\subsubsection{Unsteady jitter problem}

jitter is the variation in time between packets which causes network congestion or sometimes it changes data transmission path.

\subsubsection{Load Balancing}

load balancing is a method in which it distributes work load across multiple resources. When the load increases, network addresses a problem to server and then server causes slow traffic which results in congestion.

\section{THE IMPROVED ALGORITHM}

The analysis and comparison of above stated algorithms shown in Table 1 gives direction to design a bandwidth scheduling algorithm which suits the architecture of IEEE 802.16protocol. An important aspect of QoS which is to be considered is interference avoidance in order to minimize packet loss. QoS cannot be properly achieved without considering service priorities of the different data flow, latency, bandwidth utilization, load balancing and jitter requirements. Thus QoS makes an active research area in IEEE 802.16 since appropriate scheduling mechanisms are not specified. So, a centralized bandwidth algorithm is designed by using Karn and Nagle algorithm fundamentals which improves different performance parameters for IEEE 802.16.

\subsection{Karn's Algorithm}

In WiMax architecture at end to end protocol, TCP uses a positive acknowledgement and if retransmission occurs it results in guaranteed delivery of packets at receiver end. It measures and improves network propagation delay which results in balanced network throughput. Karn's algorithm addresses the problem of getting accurate estimates of the (RTT) Round-Trip Time messages when using TCP (Transmission Control Protocol). Accurate round trip time estimation in TCP can be difficult to calculate because of an ambiguity created by retransmitted segments. When an acknowledgement arrives for a segment, there is no indication in which retransmission is being calculated. The round trip time is measured as the difference between the time that a segment was sent and the time that its acknowledgment was returned to the sender, but ambiguity can arise at that situation because packets are retransmitted. The acknowledgment may be a response to the first transmission of the segment or to a subsequent re-transmission. Karn's Algorithm ignores retransmitted segments when updating the round trip time estimate. Round trip time estimation is based only on unambiguous acknowledgments, which are already an acknowledgment segments and sent only once. Karn's algorithm is based on two rules, which are:

Rule1: Ignored measured RTT for retransmitted packets.

Rule 2: RTO should be double after retransmission. This is known as Exponential Back Off.

The normal implementation of Karn's algorithm can lead to minor problems as well. Consider when TCP sends a segment after a sharp increase in delay. By using the prior round trip time estimation, TCP computes a timeout and retransmits a segment. If TCP never send acknowledgement to retransmitted packets, the round trip estimate time will never be updated, and TCP will continue to retransmit segments without making any change to the increased delay.
A solution to this problem is to incorporate transmission timeouts with a timer back off strategy. The timer back off strategy computes every timeout during transmission of packets. If the timer expires and causes a retransmission, TCP increases the timeout generally by a factor, 2 .

new_timeout $=2 *$ timeout

This algorithm has proven to be extremely effective in networks with high packet loss.

\subsection{Nagle's Algorithm}

This algorithm improves the efficiency of TCP network by reducing the number of packets sent over the network. Nagle reviewed "small packet problem" during congestion control in $\mathrm{TCP} / \mathrm{IP}$ network architecture. In this problem application which is running, send data in small segments known as tiny grams each of one bit size. It results in slow speed, high packet loss and congestion collapse in nodes. These limitations are overcome by using Nagle's algorithm which combine number of small transmitted data packets and sent them once during transmission. Sender must keep information of data packets until it does not receive acknowledgement. Receiver sends an instruction to sender before buffering of data. Nagle's algorithm investigates problem on FORD and ARPANET network but it does not give desirable result on client/server architecture. Nagle's algorithm can be used in one way bulk transfer, TELNET style - two way data transfer, RPC style, Real time applications.

\section{CONCLUSION}

This paper compares and analyzes different wireless scheduling algorithms. As a result, a centralized bandwidth allocation scheduling algorithm for IEEE 802.16 network is proposed which does not require any explicit information from sender for bandwidth allocation. In fact, it estimates and measures current sending and receiving rate of each flow of information between different nodes of a network. Firstly, the amount of bandwidth allocated to different services are defined which identifies the priority of these services. Secondly, the compensation mechanisms for bandwidth allocation techniques are adopted according to QoS needs for identifying bandwidth allocation on different nodes. Different issues like packet delay, limitation of data, packet loss, network throughput, load balancing on various nodes and QoS of the network are quantitatively measured and improved.

\section{ACKNOWLEDGEMENT}

Sincere thanks to SKIET, Kurukshetra, Haryana, India, for their constant encouragement.

\section{REFERENCES}

[1] Qiang Ni, Lamia Romdhani, Thierry Turletti, 2004, A Survey of QoS Enhancement for IEEE 802.11 Wireless LAN, Journal of Wireless Communication and Mobile Computing, Vol:4, Issue.5, 547-566.

[2] Ayman Khalil and Adlen Ksentini, 2005, Classification of The Uplink Scheduling Algorithm in IEEE 8O2.16, IRISA/ Université de Rennes 1 Campus Universitaire de Beaulieu 35402 Rennes Cedex.

[3] Qingwen Liu, Shengli Zhou and Georgious B.Glannakis, 2005, Cross Layer Scheduling with prescribed QoS Gurrantees in Adaptive Wireless Networking, IEEE Journal on selected area in Communication, Vol.23, No.5. 
[4] Jamshed Hasan, 2006, Security Issues of IEEE 802.16 (WiMaX), $4^{\text {th }}$ Australian Info Security Management Conference, Edith Cowan University, Perth, Western Austrailia.

[5] Alaa Z. AL-Howaide, Ahmad S. Doulat, Yaser M. Khamayseh, 2007, Performance Evaluation of Different Scheduling Algorithm in WiMAX, Department of Computer Science, Jordan University of Science and Technology, Irbid, Jordan.

[6] Mitko Bogdanoski, Pero Latkoski, Aleksandar Risteski, Borislav Popovski, 2008, IEEE 802.16 Security Issues : A Survey, Faculty of Electrical Engg. \& Information Technology, SS.Cyril and Methodius University, Macedonia, $16^{\text {th }}$ TELFOR.

[7] Jianfeng Song Jiandong Li Changle Li, 2009, A Cross Layer WiMax Scheduling Algorithm based on Genetic Algorithm, $7^{\text {th }}$ Annual Communicatiom Network and Service Research Conference, 978-0-765-3649-1/09, IEEE-DOI 10.1109/CNSR.

[8] Xiaowei MEI, Zhiyi FANG, Yunchun ZHANG, Jianfei ZHANG and Hao XIE, 2010, A WiMax QoS Oriented Bandwidth Allocation Scheduling Algorithm, School of Computer Science and Technology, Jilin University, Wireless Communication and Trusted Computing, 978-0-7695-4011-5/10, IEEE-DOI 10.1109/NS WCTC.

[9] Sonia Nazari, Hamid Beigy, 2010, Department of Information Technology Kish Campus, , Sharif University of Technology Kish Island, Iran, A New Distributed Uplink Packet Scheduling Algorithm in WiMax Network, IEEE, 978-1-4244-5824-0.

[10] Seungwoon Kim and Ikjun Yeom, 2010, TCP-Aware Uplink Scheduling for IEEEBO2.16, IEEE Communication Letters, ISSN:XX, VOL.X

[11] Ronak Farhadi, Vahid Tabataba Vakili, Shahriar Shirvani Moghada, 2011, Department of Electrical Engg., Iran University Of Science and Technology, Tehran, Iran, Performance Analysis Under The Influence of Jamming for WiMax SystemIEEE-978-1-61284-486-2/vol:11.
[12] P. saravanselvi, P.Latha, 2012, Weighted Fair Queue Scheduling Algorithm for 802.16 Wireless Network, published in International Journal of Computer Applications (IJCA), ICON 3C.

[13] Rakesh Kumar Jha, Upena D.Dalal and Vishal Wankhade, 2012, A Resource Allocation in Mobile WiMax Network: An Optimal Approach, Proceeding of the APN- Network Research Workshop.

[14] Biswapratap Singh Sahoo, 2013, IIT Kanpur, India, Performance Comparison of Packet Scheduling Algorithm for Video Traffic in LTE Cellular Network, International Journal of Mobile Communication and Telemetric (IJMNCT), Vol.3, no.3.

[15] Jyh-Ming Chen, Eric Hsiao-Kang Wu, Hsiang-Wei-Lu, Ching Hsiang Chu and Meng-Feng Tsai, 2013, Channel condition self clocked packet scheduling scheme for wireless network, Deptt. Of Computer Science and Engg., EURASIP journal on wireless communication and network, Taiwan.

[16] S.Y Amdani and M.S.Ali, 2013, An Improved Group EDF: A Real Time Disk Scheduling algorithm, International journal of computer theory and Engg. , ISSN : 6, VOL.5.

[17] Akashdeep, Karanjeet S. Kahlon, Harish Kumar, 2014 Survey of Scheduling Algorithm in IEEE 802.16PMP Network, Egyptian Informatics Journal vol.15, 25-36.

[18] R. Nandhini, N. Devarajan, 2014, Comparison for WiMax Scheduling Algorithm and Proposal Quality of Service Improvements in WiMax Networks, American Journal of Applied Sciences 11, 1546-9239, vol. 11, 816.

[19] Dheeraj Sikhwal, Dr. Rakesh Rathi, 2014, Wiamx (802.16) - A Study of Mobility and MAC Layer, IT Govt. Engg. College Ajmer, India, International Referred Journal of Engg. \& Science (IRJES), ISSN:2319-1821, vol.3, Issue:3,61-66 